\title{
Laparoscopic Repair of Vesicouterine Fistula: A Case Report and Mini-Review of the Literature
}

\author{
Sahbi Naouar ${ }^{\mathrm{a}, \mathrm{b}}$, Salem Braiek ${ }^{\mathrm{a}}$, Rafik El Kamel ${ }^{\mathrm{a}}$
}

\begin{abstract}
Vesicouterine fistula (VUF) is the rarest form of genitourinary fistulas. Despite the advantages of laparoscopy, there are few case reports showing its feasibility in the management of this rare entity. A 40-year-old woman presented to our department with urinary incontinence associated to cyclic hematuria and amenorrhea. After diagnosis of cervicovesical fistula, the laparoscopic approach was chosen. Catheterization of the fistula tract during cystoscopy at the time of laparoscopy was beneficial to localize the fistula tract and allowed meticulous dissection in the retrovesical space between the bladder and the uterus. Operating time was approximately $165 \mathrm{~min}$. The woman had no signs of recurrence after 12 months of follow-up. Laparoscopic VUF repair is an effective and safe technique with successful outcome.
\end{abstract}

Keywords: Cyclic hematuria; Laparoscopy; Vesicouterine fistula

\section{Introduction}

Vesicouterine fistula (VUF) is an anomalous communication developing between the bladder and the uterus or cervix most commonly after an iatrogenic injury during cesarean section [1]. The first case was reported by Knipe and colleagues in 1908 [2]. Among different types of genitourinary fistula, VUF is the rarest form, accounting for $1-4 \%$ of all genitourinary fistulas [3]. Conservative management with catheterization and open, laparoscopic, and robotic surgeries are the prescribed treatment options. The advantages of laparoscopy are well known; however, there are few case reports showing the feasibility of the laparoscopic repair of VUF. We report a case of laparoscopic repair of cervicovesical fistula with a successful outcome. In the light of this case and literature, the surgical

\footnotetext{
Manuscript accepted for publication November 01, 2016

aLes Aghlabides Surgical Division, Urology Department, Ibn El Jazzar Teaching Hospital, Kairouan, Tunisia

bCorresponding Author: Sahbi Naouar, Les Aghlabides Surgical Division, Urology Department, Ibn El Jazzar Teaching Hospital, Kairouan 3100, Tunisia. Email: snaouar@laposte.net
}

doi: https://doi.org/10.14740/jcs309w technique is detailed and the reported results are reviewed.

\section{Case Report}

A 40-year-old multiparous female patient presented to our urology department with urinary incontinence associated to cyclic hematuria (menouria) and amenorrhea. Her symptoms occurred 3 years after giving birth by cesarean section due to fetal distress. Physical examination done per abdomen was unremarkable except for a well-healed Pfannenstiel incision. Per vaginal exam showed normal vulva, vagina and urethral meatus. Per speculum examination revealed mildly congested cervix, and both fornices were free. Instillation of diluted methylene blue into the bladder revealed vaginal leakage. A hysterography revealed a cervicovesical fistula (Fig. 1) that was confirmed by cystoscopy showing a fistula of $8 \mathrm{~mm}$ diameter at the posterior wall of the bladder, supratrigonal in position. We chose the laparoscopic approach to treat the VUF.

Surgical technique: The procedure was performed under general anesthesia. Initially, cystoscopy was performed with bilateral ureteric catheterization using $7 \mathrm{~F}$ ureteric catheters to help identify and protect the ureters during surgical dissection. A catheter was also passed through the fistula to aid identification (Fig. 2), and then an urethral Foley catheter was placed. A primary 10 -mm port was inserted in the midline infra-umbilically. The laparoscope was introduced and two 10- and 5-mm

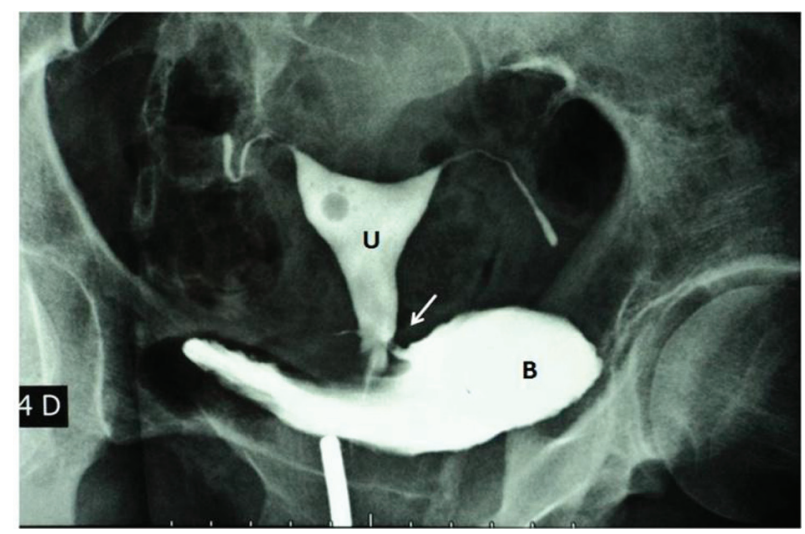

Figure 1. Hysterography reveals fistula (arrow) between uterine (U) cervix and bladder (B). 


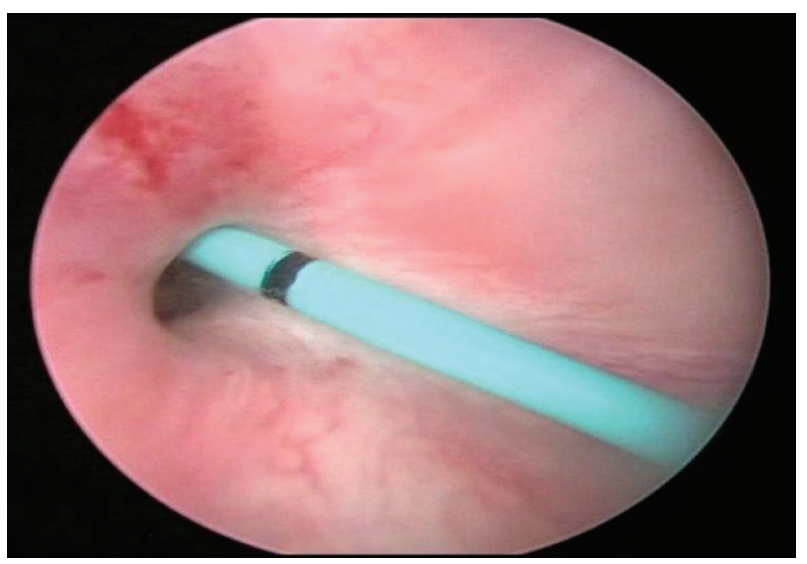

Figure 2. Cystoscopic appearance of supratrigonal and posterior fistula orifice catheterized by ureteric catheter.

secondary ports were created in both right and left iliac fossas, respectively, under laparoscopic vision. Omental adhesions in the pelvis were carefully taken down, and the uterus and bladder were identified. A malleable retractor was placed in the vagina to elevate the cervical stump and facilitate dissection. The peritoneum between the bladder and uterus was incised using a cautery, and the posterior bladder wall was found to be adherent to the uterus with dense fibrous tissue that obliterated the vesicouterine pouch. The vesicocervical space was dissected with endoscopic scissors to reveal the fistula. The catheter in the fistula came into view and was pulled into the peritoneal cavity (Fig. 3). The fistulous tract was excised, and dissection was continued $2 \mathrm{~cm}$ distally, mobilizing vagina and bladder. The defect on the posterior wall of the bladder was closed with "figure eight" sutures of 3-0 polyglactin. The cervix wound was closed with two interrupted sutures of 2-0 polyglactin. An omentum flap was anchored between the repaired ends of the fistula using two more sutures (Fig. 4).

Operating time was approximately $165 \mathrm{~min}$ and estimated blood loss was $50 \mathrm{~mL}$. No intraoperative or postoperative complications occurred. Ureter catheters were left in place for 7 days, and the bladder was drained for 15 days, after which

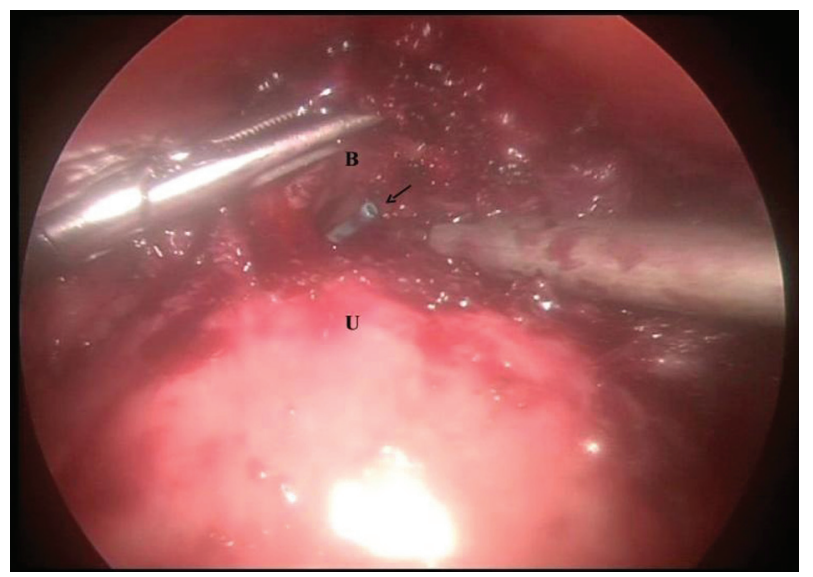

Figure 3. Laparoscopic view of vesicocervical space dissection, the ureteric catheter is seen in the opened bladder lumen (arrow).

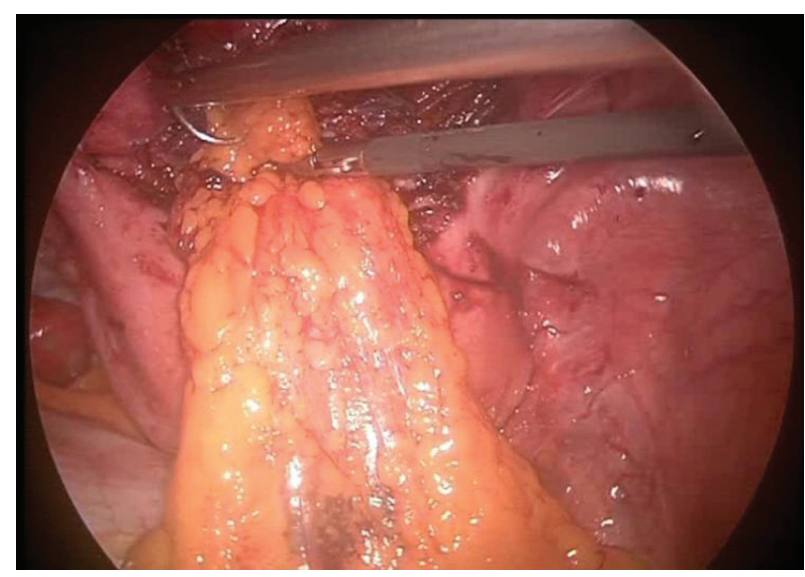

Figure 4. Omentum flap interposition between bladder and uterus.

time cystoscopy and vaginal inspection revealed a well-healed surgical site. The woman had no signs or symptoms of recurrence after 12 months of follow-up.

\section{Discussion}

VUFs are relatively uncommon compared to other urogenital fistulas. Some risk factors have been advocated for the development of VUF, such as an inadequate reflection of the bladder wall from lower uterine segment, excessive intraoperative bleeding, severe dystocia, forceps delivery, manual removal of the placenta, placenta percrata, uterine rupture, previous cesarean section or curettage $[1,3]$. Among these, cesarean section currently is the single most common cause of VUF (83-93\% of cases), and repeated cesarean sections may result in progressive devitalization and scarring of the uterus and bladder base by damaging their vascular network, thus predisposing to fistula formation [1].

Patients with VUF can have various clinical presentations. Usually immediate features occur when there is direct injury to the bladder during surgery. Patients can have early hematuria and/or urinary leakage [4]. Delayed presentation can occur when there is a progressive devitalization of the posterior wall of the bladder and patients often have symptoms of urinary leakage from the vagina, if the cervix is incompetent, cyclic hematuria (menouria), amenorrhea, infertility or first trimester abortion [5]. A classification of VUF based on the routes of menstrual flow has been proposed by Jozwik and Jozwik that divides VUF into three types. Type I, characterized by the triad of amenorrhea, menouria without urinary incontinence has been known as Youssef's syndrome. Type II is associated with dual menstrual flow via both the bladder and vagina. Type III is associated with pure urine incontinence [6]. The sphincteric effect of cervical isthmus maintains sufficient pressure to prevent urinary leakage. Our patient qualified into type II VUF.

Confirming the diagnosis of a VUF may be challenging. Hysterography and cystoscopy remain the "gold standard" in the diagnosis of VUF, but these modalities may not reveal the fistula if it is small [7]. If the findings are inconclusive, the diagnosis can be confirmed by methylene blue instilled into the bladder to demonstrate leakage from the cervix on colposcopy. 
Additional diagnostic procedures include contrast-enhanced computed tomography (CT) and magnetic resonance imaging (MRI) procedures. MRI can delineate the fistula tract and its relation to the bladder and uterus, because of differences in the intensity of the fistula tract and the uterus in T2-weighted scans; therefore, it is thought to be a superior non-invasive imaging modality [7].

Treatment options depend upon fistula size, time of presentation and symptoms. Continuous bladder drainage is the conservative management option for patients who are in the early postpartum phase with a small fistula. The success rate reported with conservative management is less than 5\% [3]. Hormonal therapy consists of induction of artificial amenorrhea with gonadotropin-releasing hormone analogues or oral contraceptive pills to prevent blood drainage through the fistula tract [8]. It is particularly useful in treatment of small VUFs presenting with Youssef's syndrome.

Surgery is the most effective treatment modality for VUF with different approaches including vaginal, transvesical, transperitoneal and laparoscopic procedures. Open technique remains the mainstay with good results $[3,7]$. The surgical principles are liberal excision of scar tissues around the fistula, tension-free closure of the wound, and the interposition of an omental flap, while in the literature use of free adipose tissue grafts prepared from abdominal fat has been described. Interrupted, absorbable sutures should be used to avoid necrosis. The repaired region must have a good blood supply and bladder should be drained postoperatively [9].

On the other hand, the laparoscopic technique allows for transabdominal access with improvement in visualization and magnification resulting in excellent exposure to the vesicouterine pouch and retrovesical space [9, 10]. The first case of laparoscopic repair of VUF was reported by Miklos in 1999 [9]. It has proved advantage in terms of lower pain, quicker convalescence, shorter hospital stay, and better cosmetic results, with success rates similar to those of open surgery $[9,10]$. Regardless of mode of access (laparoscopy vs. laparotomy), the same surgical principles would be used to repair the fistula. In our case, catheterization of the fistula tract during cystoscopy at the time of laparoscopy was beneficial to localize the fistula tract, and allowed meticulous dissection in the retrovesical space between the bladder and the uterus, and resection of the fistula tract with minimal manipulation of the bladder.

\section{Conclusion}

Laparoscopic VUF repair is an effective and safe technique with successful outcome. Technically, it provides better visualization through magnification, but is more difficult to learn, as is intracorporeal suturing. Another difficult technical challenge is treating sufficient patients to stay proficient.

\section{Conflicts of Interest}

None.

\section{Funding Source}

None.

\section{References}

1. Yossepowitch O, Baniel J, Livne PM. Urological injuries during cesarean section: intraoperative diagnosis and management. J Urol. 2004;172(1):196-199.

2. Iloabachie GC, Njoku O. Vesico-uterine fistula. Br J Urol. 1985;57(4):438-439.

3. Porcaro AB, Zicari M, Zecchini Antoniolli S, Pianon R, Monaco C, Migliorini F, Longo M, et al. Vesicouterine fistulas following cesarean section: report on a case, review and update of the literature. Int Urol Nephrol. 2002;34(3):335-344.

4. Mercader VP, McGuckin JF, Jr., Caroline DF, Chatwani A, Seidmon EJ. CT of vesicocorporeal fistula with menouria: a complication of uterine biopsy. J Comput Assist Tomogr. 1995;19(2):324-326.

5. Lenkovsky Z, Pode D, Shapiro A, Caine M. Vesicouterine fistula: a rare complication of cesarean section. J Urol. 1988;139(1):123-125.

6. Jozwik M. Clinical classification of vesicouterine fistula. Int J Gynaecol Obstet. 2000;70(3):353-357.

7. Shanmugasundaram R, Gopalakrishnan G, Kekre NS. Youssef's syndrome: Is there a better way to diagnose? Indian J Urol. 2008;24(2):269-270.

8. Hemal AK, Wadhwa SN, Kriplani A, Hemal U. Youssef's syndrome: an appraisal of hormonal treatment. Urol Int. 1994;52(1):55-57.

9. Miklos JR. Laparoscopic treatment of vesicouterine fistula. J Am Assoc Gynecol Laparosc. 1999;6(3):339-341.

10. Chibber PJ, Shah HN, Jain P. Laparoscopic O'Conor's repair for vesico-vaginal and vesico-uterine fistulae. BJU Int. 2005;96(1):183-186. 